\title{
MIXED HODGE-RIEMANN BILINEAR RELATIONS AND M-POSITIVITY
}

\author{
JIAN XIAO
}

\begin{abstract}
Motivated by our previous work on Hodge-index type inequalities, we give a form of mixed Hodge-Riemann bilinear relation by using the notion of $m$-positivity, whose proof is an adaptation of the works of Timorin and Dinh-Nguyên. This mixed Hodge-Riemann bilinear relation holds with respect to mixed polarizations in which some satisfy particular positivity condition, but could be degenerate along some directions. In particular, it applies to fibrations of compact Kähler manifolds.
\end{abstract}

\section{Contents}

1. Introduction 1

2. Preliminaries 3

3. Proof of the main result 5

4. Further remarks $\quad 8$

References $\quad 9$

\section{INTRODUCTION}

1.1. The classical and mixed HRR. Let $X$ be a compact Kähler manifold of dimension $n$, and let $\omega$ be a Kähler class on $X$. Let $0 \leq p, q \leq p+q \leq n$ be integers. Denote $\Omega=\omega^{n-p-q}$, which is a strictly positive class in $H^{n-p-q, n-p-q}(X, \mathbb{C})$. Associated to $\Omega$, one could define the following quadratic form $Q$ on $H^{p, q}(X, \mathbb{C})$ :

$$
Q(\Phi, \Psi)=i^{q-p}(-1)^{(p+q)(p+q+1) / 2} \Omega \cdot \Phi \cdot \bar{\Psi} .
$$

The classical Hodge-Riemann bilinear relation theorem (HRR) (see e.g. [Dem12], [Voi07]) states that $Q$ is positive definite on the primitive subspace $P^{p, q}(X, \mathbb{C})$, which is defined as follows:

$$
P^{p, q}(X, \mathbb{C})=\left\{\Phi \in H^{p, q}(X, \mathbb{C}) \mid \Omega \cdot \omega \cdot \Phi=\omega^{n-p-q+1} \cdot \Phi=0\right\} .
$$

As a corollary, one could get the classical Hard Lefchetz theorem (HL), i.e., the linear map

$$
\begin{aligned}
& \Omega: H^{p, q}(X, \mathbb{C}) \rightarrow H^{n-q, n-p}(X, \mathbb{C}), \\
& \Phi \mapsto \Omega \cdot \Phi
\end{aligned}
$$

is an isomorphism. Furthermore, by the classical HRR, one could also get the Lefchetz decomposition theorem (LD), that is, there is an orthogonal decomposition

$$
H^{p, q}(X, \mathbb{C})=P^{p, q}(X, \mathbb{C}) \oplus \omega \wedge H^{p-1, q-1}(X, \mathbb{C})
$$

with respect to $Q$, with the convention that $H^{p-1, q-1}(X, \mathbb{C})=\{0\}$ if either $p=0$ or $q=0$.

In [DN06, Cat08] (see also [Gro90, Tim98]), the classical HRR is greatly generalized to the mixed situation. More precisely, let $\omega_{1}, \ldots, \omega_{n-p-q+1}$ be Kähler classes on $X$. Denote $\Omega=\omega_{1} \cdot \ldots \cdot \omega_{n-p-q}$. We call $\Phi \in H^{p, q}(X, \mathbb{C})$ primitive with respect to $\Omega \cdot \omega_{n-p-q+1}$, if

$$
\Omega \cdot \omega_{n-p-q+1} \cdot \Phi=0 .
$$

The mixed HRR states that the corresponding quadratic form $Q$ defined by $\Omega$ is positive definite on the subspace of primitive classes. This mixed HRR then implies the mixed versions of HL and LD.

The reader can find some applications of this mixed HRR in complex geometry in [DS04,DS05]. One can also find some related topics and applications in the other contexts in [EW14], [Wil16], [AHK18], [McM93], [Tim99], [dCM02] and the references therein. 
1.2. The main result. Our aim is to weaken the positivity of $(1,1)$ classes, which we also call polarizations, in the definition of $\Omega$ such that the HRR still holds. The key positivity notion is $m$-positivity.

Let $\omega$ be a reference Kähler metric on $X$ of dimension $n$. Then a real smooth $(1,1)$ form $\widehat{\alpha}$ is called $m$-positive $(1 \leq m \leq n)$ with respect to $\omega$, if

$$
\widehat{\alpha}^{k} \wedge \omega^{n-k}>0
$$

holds for any $1 \leq k \leq m$ and any point on $X$. It is well-known that $\widehat{\alpha}$ is $n$-positive if and only if it is strictly positive. This kind of positivity plays an important role in the study of Hessian equations, as it gives the natural solution set of such PDEs. A cohomology class $\alpha \in H^{1,1}(X, \mathbb{R})$ is called $m$-positive with respect to the metric $\omega$, if it has a smooth representative which is $m$-positive in the pointwise sense.

In our previous work [Xia18], inspired by the Hodge-index type inequalities obtained by complex Hessian equations, we proved a Hodge-index type theorem for classes of type $(1,1)$ by using $m$ positivity and Garding's theory of hyperbolic polynomials. More precisely, let $\alpha_{1}, \ldots, \alpha_{m-1}$ be $m$ positive classes, and denote

$$
\Omega=\omega^{n-m} \cdot \alpha_{1} \cdot \ldots \cdot \alpha_{m-2},
$$

then the HRR holds with respect to $\Omega$, where the primitive subspace is defined by $\Omega \cdot \alpha_{m-1}$. This is the main motivation of our work.

We generalize this result to arbitrary $(p, q)$ classes, by assuming further that the $\alpha_{j}$ are semipositive. Note that: if an $(1,1)$ form is semipositive, then it is $m$-positive with respect to $\omega$ if and only if it has at least $m$ positive eigenvalues. Thus our positivity assumption is that every $\alpha_{j}$ has a semipositive smooth representative, which has at least $m$ positive eigenvalues at every point of $X$.

Theorem A. Let $X$ be a compact Kähler manifold of dimension n, and let $\omega$ be a Kähler class on $X$. Let $p, q, m$ be integers such that $0 \leq p, q \leq p+q \leq m \leq n$. Let $\alpha_{1}, \ldots, \alpha_{m-p-q+1} \in H^{1,1}(X, \mathbb{R})$. Assume that every $\alpha_{j}$ has a smooth representative which is semipositive and has at least $m$ positive eigenvalues at every point. Denote $\Omega=\omega^{n-m} \cdot \alpha_{1} \cdot \alpha_{2} \cdot \ldots \cdot \alpha_{m-p-q}$. Let

$$
P^{p, q}(X, \mathbb{C})=\left\{\Phi \in H^{p, q}(X, \mathbb{C}) \mid \Omega \cdot \alpha_{m-p-q+1} \cdot \Phi=0\right\}
$$

be the primitive subspace defined by $\Omega \cdot \alpha_{m-p-q+1}$. Then

- (HRR) the quadratic form

$$
Q(\Phi, \Psi)=i^{q-p}(-1)^{(p+q)(p+q+1) / 2} \Omega \cdot \Phi \cdot \bar{\Psi}
$$

is positive definite on $P^{p, q}(X, \mathbb{C})$.

This implies that

- $(H L)$ the map

$$
\Omega: H^{p, q}(X, \mathbb{C}) \rightarrow H^{n-q, n-p}(X, \mathbb{C}), \Phi \mapsto \Omega \cdot \Phi
$$

is an isomorphism.

- (LD) the space $H^{p, q}(X, \mathbb{C})$ has an orthogonal decomposition

$$
H^{p, q}(X, \mathbb{C})=P^{p, q}(X, \mathbb{C}) \oplus \alpha_{m-p-q+1} \wedge H^{p-1, q-1}(X, \mathbb{C})
$$

with respect to $Q$, and $\operatorname{dim} P^{p, q}(X, \mathbb{C})=\operatorname{dim} H^{p, q}(X, \mathbb{C})-\operatorname{dim} H^{p-1, q-1}(X, \mathbb{C})$, where we use the convention that $H^{p-1, q-1}(X, \mathbb{C})=\{0\}$ if either $p=0$ or $q=0$.

Remark 1.1. In general, the HRR, HL and LD theorems are not true if $\Omega$ is an arbitrary class in $H^{n-p-q, n-p-q}(X, \mathbb{C})$, even if the class has a smooth strictly positive representative (see e.g. [BS02, Section 9], [DN13, Remark 2.9]). Thus Theorem A provides a sufficient condition on $\Omega$ such that HRR, HL and LD hold true. 
1.3. Relative HRR. Interesting examples are given by the holomorphic fibrations between compact Kähler manifolds.

Corollary A. Let $f: X \rightarrow Y$ be a holomorphic submersion from a compact Kähler manifold of dimension $n$ to a compact Kähler manifold of dimension $m$. Let $p, q, m$ be non-negative integers such that $p+q \leq m$. Assume that $\omega_{X}$ is a Kähler class on $X$ and $\omega_{Y_{1}}, \ldots, \omega_{Y_{m-p-q+1}}$ are Kähler classes on $Y$, then the HRR holds with respect to

$$
\Omega_{X, Y}=\omega_{X}^{n-m} \cdot f^{*} \omega_{Y_{1}} \cdot \ldots \cdot f^{*} \omega_{Y_{m-p-q}},
$$

where the primitive space is defined by $\Omega_{X, Y} \cdot f^{*} \omega_{Y_{m-p-q+1}}$.

This is true because every $f^{*} \omega_{Y_{k}}$ is $m$-positive with respect to some Kähler metric on $X$ and semipositive. In some sense, this can be seen as a relative version of the classical and mixed HRR.

Remark 1.2. When $f$ is just a surjective holomorphic map, the $f^{*} \omega_{Y_{j}}$ is $m$-positive at generic points, that is, it has a smooth representative which is $m$-positive on a Zariski open set. In this general setting, we are not quite sure what kind of condition could be proposed to $f$ such that the "relative" HRR holds if and only if the condition on $f$ holds. Note that the relative HRR or HL is not true for a general map. Nevertheless, for general holomorphic maps, see Section 4.2 for some discussions. We intend to address this in a future work.

To our knowledge, there are only some known results (see [dCM02]) when $f: X \rightarrow Y$ is a semi-small map between projective varieties and the $\omega_{Y_{j}}$ are given by the same ample line bundle on $Y$. For this particular case, $f$ is generically finite, thus $n=m$.

1.4. About the proof. To prove the main result, we mainly follow the approach of Timorin [Tim98] and Dinh-Nguyên [DN06,DN13]. First, we apply Timorin's inductive argument to establish the linear version of our HRR. To this end, we need to study the positivity of restrictions of $m$-positive forms. This is the only place where we need to assume further that the $\alpha_{j}$ are semipositive, not only $m$ positive. Once the linear HRR is proved, then we could apply the $d d^{c}$-method to reduce the global case to the local case.

\section{Preliminaries}

2.1. Restriction of $m$-positive forms. In this section, we consider the problem on positivity of the restrictions of $m$-positive forms. Let $\Lambda_{\mathbb{R}}^{1,1}\left(\mathbb{C}^{n}\right)$ be the space of real $(1,1)$ forms on $\mathbb{C}^{n}$ with constant coefficients.

Lemma 2.1. Let $\omega$ be a Kähler metric on $\mathbb{C}^{n}$ with constant coefficients. Assume that $\alpha \in \Lambda_{\mathbb{R}}^{1,1}\left(\mathbb{C}^{n}\right)$ is m-positive with respect to $\omega$. Then

- for any $1 \leq k \leq m-1$ and any hyperplane $H \subset \mathbb{C}^{n}$, we have $\alpha_{\mid H}^{k} \wedge \omega_{\mid H}^{n-k-1}>0$ on $H$.

- for $k=m$, if we assume further that $\alpha$ is semipositive, then there is a proper subspace $S(\alpha)$ such that for any $v \in \mathbb{C}^{n} \backslash S(\alpha)$, we have $\alpha_{\mid H_{v}}^{m} \wedge \omega_{\mid H_{v}}^{n-m-1}>0$ on $H_{v}$, where

$$
H_{v}=\left\{z \in \mathbb{C}^{n} \mid v \cdot z=0\right\}
$$

is the hyperplane defined by $v$.

Proof. Let $a=\left(a_{1}, \ldots, a_{n}\right)$ be a non-zero vector in $\mathbb{C}^{n}$. Let $H_{a}$ be the hyperplane defined by $a$. Denote the equation of $H_{a}$ by the same notation $H_{a}(z)=a \cdot z$.

Since $a \neq 0$, we could find linear functions $w_{1}, \ldots, w_{n-1}$ of $z$ such that $\left(w_{1}, \ldots, w_{n-1}, H_{a}\right)$ give a new coordinate system of $\mathbb{C}^{n}$. Write $\alpha=\alpha_{0}+\beta_{0}$, where $\beta_{0}$ is the sum of $(1,1)$ forms containing either $d H_{a}$ or $d \overline{H_{a}}$. Then $\alpha_{0}=\alpha_{\mid H_{a}}$. Similarly, we write $\omega=\omega_{\mid H_{a}}+\beta_{1}$. It is easy to see that

$$
\alpha^{k} \wedge \omega^{n-k-1} \wedge i d H_{a} \wedge d \overline{H_{a}}=\alpha_{\mid H_{a}}^{k} \wedge \omega_{\mid H_{a}}^{n-k-1} \wedge i d H_{a} \wedge d \overline{H_{a}} .
$$

In particular, if we denote $V=i^{n-1} d w_{1} \wedge d \bar{w}_{1} \wedge \ldots \wedge d w_{n-1} \wedge d \bar{w}_{n-1}$, then (1) yields

$$
\frac{\alpha^{k} \wedge \omega^{n-k-1} \wedge i d H_{a} \wedge d \overline{H_{a}}}{V \wedge i d H_{a} \wedge d \overline{H_{a}}}=\frac{\alpha_{\mid H_{a}}^{k} \wedge \omega_{\mid H_{a}}^{n-k-1}}{V} .
$$


For simplicity, we just write (2) as

$$
\alpha^{k} \wedge \omega^{n-k-1} \wedge i d H_{a} \wedge d \overline{H_{a}}=\alpha_{\mid H_{a}}^{k} \wedge \omega_{\mid H_{a}}^{n-k-1} .
$$

We first prove the first statement. For $1 \leq k \leq m-1$, we can write

$$
\alpha^{k} \wedge \omega^{n-k-1} \wedge i d H_{a} \wedge d \overline{H_{a}}=\omega^{n-m} \wedge \omega^{m-k-1} \wedge \alpha^{k} \wedge \wedge i d H_{a} \wedge d \overline{H_{a}} .
$$

Since $\omega, \alpha$ are $m$-positive with respect to $\omega$, by the theory of hyperbolic polynomials (see e.g. [Gar59], [Hor94, Chapter 2], [Xia18, Lemma 3.8]), $\alpha^{k} \wedge \omega^{n-k-1}$ is strictly positive. Thus for any non-zero semipositive $(1,1)$ form $\beta$,

$$
\alpha^{k} \wedge \omega^{n-k-1} \wedge \beta>0 .
$$

Letting $\beta=i d H_{a} \wedge d \overline{H_{a}}$ and using (3) finish the proof.

Next we consider the second statement. Assume that

$$
\alpha^{m} \wedge \omega^{n-m-1}=\sum_{i, j} \Phi_{i j} d \widehat{z_{i} \wedge d} \bar{z}_{j},
$$

where $d \widehat{z_{i} \wedge d} \bar{z}_{j}$ is the $(n-1, n-1)$ form omitting $d z_{i}, d \bar{z}_{j}$ such that

$$
d \widehat{z_{i} \wedge d} \bar{z}_{j} \wedge i d z_{i} \wedge d \bar{z}_{j}>0
$$

After dividing a volume form, we have

$$
\alpha^{m} \wedge \omega^{n-m-1} \wedge i d H_{a} \wedge d \overline{H_{a}}=\sum_{i j} \Phi_{i j} a_{i} \overline{a_{j}} .
$$

As we have assumed further that $\alpha$ is semipositive, the Hermitian matrix $\left[\Phi_{i j}\right]$ is semipositive and non-zero. This implies that the set

$$
S(\alpha)=\left\{\left(a_{1}, \ldots, a_{n}\right) \in \mathbb{C}^{n} \mid \sum_{i j} \Phi_{i j} a_{i} \overline{a_{j}}=0\right\}
$$

is a proper linear subspace of $\mathbb{C}^{n}$. Thus for any $v \in \mathbb{C}^{n} \backslash S(\alpha)$, we have

$$
\alpha^{m} \wedge \omega^{n-m-1} \wedge i d H_{v} \wedge d \overline{H_{v}}>0 .
$$

By (3), this is equivalent to

$$
\alpha_{\mid H_{v}}^{m} \wedge \omega_{\mid H_{v}}^{n-m-1}>0
$$

This finishes the proof of the second statement.

Remark 2.2. Take a coordinate system such that $\omega=i \sum_{j=1}^{n} d z_{j} \wedge d \bar{z}_{j}, \alpha=i \sum_{j=1}^{n} \lambda_{j} d z_{j} \wedge d \bar{z}_{j}$. If $\alpha$ is only $m$-positive with respect to $\omega$, then (3) implies that $\alpha_{\mid H_{e}}$ is $m$-positive with respect to $\omega_{\mid H_{e}}$ for any

$$
e \in\left\{\left(a_{1}, \ldots, a_{n}\right) \mid a_{i}= \pm 1\right\} .
$$

To verify this, we only need to observe that

$$
\alpha^{k} \wedge \omega^{n-k-1} \wedge i d H_{e} \wedge d \overline{H_{e}}=\alpha^{k} \wedge \omega^{n-k} .
$$

In particular, this shows that there is an open set $\mathcal{O}_{1}$ (independent of $\alpha$ ) of hyperplanes containing all the $H_{e}$ such that, for any $H \in \mathcal{O}_{1}, \alpha_{\mid H}$ is $m$-positive with respect to $\omega_{\mid H}$. By induction, this yields that there is an open set $\mathcal{O}_{m}$ (independent of $\alpha$ ) of linear subspaces of dimension $m$ such that, for any $V \in \mathcal{O}_{m}, \alpha_{\mid V}$ is Kähler on $V$. 
2.2. Existence of orthogonal bases. In order to prove the linear HRR by induction, we need the following existence result.

Lemma 2.3. Let $H_{v_{1}}, \ldots, H_{v_{k}} \subset \mathbb{C}^{n}$ be hyperplanes, then there is an orthonormal basis $\left(e_{1}, \ldots, e_{n}\right)$ such that every $e_{i} \in \mathbb{C}^{n} \backslash \cup_{i=1}^{k} H_{v_{i}}$.

Proof. We first take

$$
e_{1} \in \mathbb{C}^{n} \backslash\left(\cup_{i=1}^{k} H_{v_{i}} \bigcup \cup_{i=1}^{k} \mathbb{C} v_{i}\right) .
$$

Then for every $i, H_{v_{i}} \cap H_{e_{1}}$ is a hyperplane in $H_{e_{1}}$. By induction, there is an orthonormal basis $\left(e_{2}, \ldots, e_{n}\right)$ such that every

$$
e_{i} \in H_{e_{1}} \backslash \bigcup_{i=1}^{k}\left(H_{v_{i}} \cap H_{e_{1}}\right) .
$$

The vectors $e_{1}, \ldots, e_{n}$ give the desired basis.

\section{Proof of the main Result}

3.1. The local case. We first adapt the arguments of Timorin [Tim98] to give a form of HRR in the linear case. Roughly speaking, Timorin's proof goes by induction:

- assume that HRR holds for $\operatorname{dim} \leq n-1$, then it can be used to prove HL for $\operatorname{dim}=n$;

- the HL for $\operatorname{dim}=n$ yields HRR for $\operatorname{dim}=n$.

Theorem 3.1. Let $\omega \in \Lambda_{\mathbb{R}}^{1,1}\left(\mathbb{C}^{n}\right)$ be a Kähler metric on $\mathbb{C}^{n}$. Let $p, q$ be arbitrary integers satisfying $0 \leq p, q \leq p+q \leq m \leq n$. Assume that $\alpha_{1}, \ldots, \alpha_{m-p-q+1} \in \Lambda_{\mathbb{R}}^{1,1}\left(\mathbb{C}^{n}\right)$ are $m$-positive with respect to $\omega$ and semipositive. Denote

$$
\Omega=\omega^{n-m} \wedge \alpha_{1} \wedge \ldots \wedge \alpha_{m-p-q}
$$

Then the HRR holds with respect to $\Omega$, where the primitive space is defined by $\Omega \wedge \alpha_{m-p-q+1}$.

In the linear case, the quadratic form $Q$ is defined by $Q(\Phi, \Psi)=\Omega \wedge \Phi \wedge \bar{\Psi} / \Gamma$, where $\Gamma$ is a fixed volume form of $\mathbb{C}^{n}$. Note that when $n=m$, Theorem 3.1 is exactly the linear version of mixed HRR recalled in the introduction.

Remark 3.2. It is clear that the linear version of HRR is equivalent to the HRR on a compact complex torus.

We denote the space of complex $(p, q)$ forms on $\mathbb{C}^{n}$ with constant coefficients by $\Lambda^{p, q}$.

As stated above, Theorem 3.1 will be proved by induction on dimensions.

Lemma 3.3. Assume Theorem 3.1 holds for $\operatorname{dim}=n-1$. Then the $H L$ holds for $\operatorname{dim}=n$, i.e., $\Omega: \Lambda^{p, q} \rightarrow \Lambda^{n-q, n-p}$ is an isomorphism.

Proof. When $p+q=m$ or $m=n$, it is the classical HL recalled in the introduction. Thus we only need to consider the case when $p+q<m<n$.

Without loss of generalities, we can assume $\omega=i \sum_{j=1}^{n} d z_{j} \wedge d \bar{z}_{j}$.

We only need to prove that the map defined by $\Omega$ is injective. Assume that $\Phi \in \Lambda^{p, q}$ satisfies $\Omega \wedge \Phi=0$, then for any hyperplane $H$,

$$
\omega_{\mid H}^{n-m-1} \wedge \omega_{\mid H} \wedge \alpha_{1 \mid H} \wedge \ldots \wedge \alpha_{m-p-q \mid H} \wedge \Phi_{\mid H}=0 .
$$

This implies that $\Phi_{\mid H}$ is primitive on $H$, where $\alpha_{m-p-q+1}=\omega_{\mid H}$ on $H$.

By Lemma 2.1, for every general hyperplane $H=H_{v}$, where

$$
v \in \mathbb{C}^{n} \backslash \bigcup_{j=1}^{m-p-q} S\left(\alpha_{j}\right),
$$

the restrictions $\alpha_{j \mid H}$ are $m$-positive with respect to $\omega_{\mid H}$. The restrictions $\alpha_{j \mid H}$ are also semipositive.

Denote $c=i^{q-p}(-1)^{(p+q)(p+q+1) / 2}$. By induction, the HRR holds in lower dimensions, thus

$$
Q_{H}\left(\Phi_{\mid H}, \Phi_{\mid H}\right)=c \omega_{\mid H}^{n-m-1} \wedge \alpha_{1 \mid H} \wedge \ldots \wedge \alpha_{m-p-q \mid H} \wedge \Phi_{\mid H} \wedge \overline{\Phi_{\mid H}} \geq 0 .
$$


By the same argument for (1), (4) is equivalent to

$$
c \omega^{n-m-1} \wedge \alpha_{1} \wedge \ldots \wedge \alpha_{m-p-q} \wedge \Phi \wedge \bar{\Phi} \wedge i d H \wedge d \bar{H} \geq 0 .
$$

Note that every $S\left(\alpha_{j}\right)$ is contained in a hyperplane, by Lemma 2.3 we can take an orthonormal basis $e_{1}, \ldots, e_{n}$ such that every

$$
e_{k} \in \mathbb{C}^{n} \backslash \bigcup_{j=1}^{m-p-q} S\left(\alpha_{j}\right)
$$

Applying (5) to $H_{e_{j}}$ and taking the sum over $j$ imply

$$
c \omega^{n-m} \wedge \alpha_{1} \wedge \ldots \wedge \alpha_{m-p-q} \wedge \Phi \wedge \bar{\Phi} \geq 0,
$$

by using that

$$
i \sum_{j} d H_{e_{j}} \wedge d \overline{H_{e_{j}}}=\omega .
$$

By the assumption $\Omega \wedge \Phi=0$, (6) is an equality, thus

$$
Q_{H_{e_{j}}}\left(\Phi_{\mid H_{e_{j}}}, \Phi_{\mid H_{e_{j}}}\right)=0
$$

for every $j$. By induction, this yields that $\Phi_{\mid H_{e_{j}}}=0$ for every $j$.

We claim that this implies $\Phi=0$.

Without loss of generalities, we can assume $H_{e_{j}}(z)=z_{j}$. The claim can be proved by contradiction. Assume

$$
\Phi=\sum_{|I|=p,|J|=q} \Phi_{I J} d z_{I} \wedge d \bar{z}_{J} \neq 0,
$$

then there is a term $\Phi_{I J} d z_{I} \wedge d \bar{z}_{J} \neq 0$. Since $p+q<m<n$, there must exist some $j$ such that the multi-indexes $I, J$ do not contain $j$. This implies $\Phi_{\mid H_{e_{j}}} \neq 0$, a contradiction.

This finishes the proof of the lemma.

Remark 3.4. Without the assumption on the semipositivity of $\alpha_{j}$, we associate $\alpha_{j}$ to the following open set

$$
P\left(\alpha_{j}\right)=\left\{v \in \mathbb{C}^{n} \mid \alpha_{j}^{m} \wedge \omega^{n-m-1} \wedge i d H_{v} \wedge d \overline{H_{v}}>0\right\} .
$$

Then by Lemma 2.1, it is clear that $\alpha_{j \mid H_{v}}$ is $m$-positive with respect to $\omega_{\mid H_{v}}$ on $H_{v}$ for every $v \in P\left(\alpha_{j}\right)$. It is unclear to us whether the intersection

$$
\bigcap_{j=1}^{m-p-q} P\left(\alpha_{j}\right)
$$

always contains an othonormal basis. If this was true, then we could apply the induction as above and remove the semipositivity assumption. By Lemma 2.3, this holds when the $\alpha_{j}$ are also semipositive.

Lemma 3.5. In the same setting as Theorem 3.1, assume that the $H L$ holds for $\operatorname{dim}=n$, then the quadratic form $Q$ defined by $\Omega=\omega^{n-m} \wedge \alpha_{1} \wedge \ldots \wedge \alpha_{m-p-q}$ is non-degenerate on $\Lambda^{p, q}$.

Proof. This follows directly from Lemma 3.3.

Another consequence of the HL in dimension $n$ is the following LD.

Lemma 3.6. In the same setting as Theorem 3.1, assume that the $H L$ holds for $\operatorname{dim}=n$, then the space $\Lambda^{p, q}$ has a $Q$-orthogonal direct sum decomposition

$$
\Lambda^{p, q}=P^{p, q} \oplus\left(\alpha_{m-p-q+1} \wedge \Lambda^{p-1, q-1}\right),
$$

where we use the convention that $\Lambda^{p-1, q-1}=\{0\}$ when $p=0$ or $q=0$. Moreover,

$$
\operatorname{dim} P^{p, q}=\operatorname{dim} \Lambda^{p, q}-\operatorname{dim} \Lambda^{p-1, q-1},
$$

which is independent of $\Omega$ and $\alpha_{m-p-q+1}$. 
Proof. By the assumption, the map $\Omega \wedge \alpha_{m-p-q+1}^{2}: \Lambda^{p-1, q-1} \rightarrow \Lambda^{n-q+1, n-p+1}$ is an isomorphism. Thus, the map $\alpha_{m-p-q+1}: \Lambda^{p-1, q-1} \rightarrow \Lambda^{p, q}$ is injective, and

$$
P^{p, q} \cap\left(\alpha_{m-p-q+1} \wedge \Lambda^{p-1, q-1}\right)=\{0\} .
$$

We also get that

$$
\Omega \wedge \alpha_{m-p-q+1}: \Lambda^{p, q} \rightarrow \Lambda^{n-q+1, n-p+1}
$$

is an isomorphism when restricted to $\alpha_{m-p-q+1} \wedge \Lambda^{p-1, q-1}$. The kernel of $\Omega \wedge \alpha_{m-p-q+1}$ is exactly given by $P^{p, q}$. On the hand, note that

$$
\operatorname{dim}\left(\alpha_{m-p-q+1} \wedge \Lambda^{p-1, q-1}\right)=\operatorname{dim} \Lambda^{p-1, q-1}=\operatorname{dim} \Lambda^{n-q+1, n-p+1} .
$$

Thus, $\operatorname{dim} \Lambda^{p, q}=\operatorname{dim} P^{p, q}+\operatorname{dim} \alpha_{m-p-q+1} \wedge \Lambda^{p-1, q-1}$.

The orthogonality follows directly from the definition of $P^{p, q}$.

Proof of Theorem 3.1. Assume that the HRR holds in dim $=n-1$, then by Lemma 3.3 the HL holds in $\operatorname{dim}=n$. Thus we could apply Lemmas 3.5 and 3.6. Theorem 3.1 will follow from a homotopy argument as in [Tim98]. Consider the deformation

$$
\Omega_{t}=\omega^{n-m} \wedge\left((1-t) \alpha_{1}+t \omega\right) \wedge \ldots \wedge\left((1-t) \alpha_{m-p-q}+t \omega\right), 0 \leq t \leq 1 .
$$

The primitive space $P_{t}^{p, q}$ is defined by $\Omega_{t} \wedge\left((1-t) \alpha_{m-p-q+1}+t \omega\right)$. Note that every $(1-t) \alpha_{1}+t \omega$ is $m$-positive and semipositive, thus all the quadratic forms $Q_{t}$ are non-degenerate and all the primitive subspaces $P_{t}^{p, q}$ have the same dimension. When $t=1, Q_{1}$ is positive definite by the classical HRR. Thus $Q_{0}$ is also positive definite, since $Q_{t}$ never becomes degenerate in the course of deformation.

Therefore, the HRR holds in $\operatorname{dim}=n$. This finishes the proof.

The following local estimate is important when reducing the global case to the local case.

Recall that the space $\Lambda^{p, q}$ admits an inner product defined by

$$
\langle\Phi, \Psi\rangle=\sum_{I, J} \Phi_{I J} \overline{\Psi_{I J}}
$$

where $\Phi=\sum_{I, J} \Phi_{I J} d z_{I} \wedge d \bar{z}_{J}, \Psi=\sum_{I, J} \Psi_{I J} d z_{I} \wedge d \bar{z}_{J}$. The norm is given by $\|\Phi\|^{2}=\langle\Phi, \Phi\rangle$.

Lemma 3.7. Using the same notations as Theorem 3.1, there are positive constants $c_{1}, c_{2}$ such that

$$
\|\Phi\|^{2} \leq c_{1} Q(\Phi, \Phi)+c_{2}\left\|\Omega \wedge \alpha_{m-p-q+1} \wedge \Phi\right\|^{2}, \forall \Phi \in \Lambda^{p, q} .
$$

Proof. By Theorem 3.1 and Lemma 3.6, the proof is the same as that of [DN06, Proposition 2.2] (see also [DN13, Proposition 2.8]).

3.2. The global case. Following [DN06, DN13], the global case (Theorem A) can be reduced to the local case (Theorem 3.1) by solving a $d d^{c}$-equation.

We denote the space of smooth complex $(p, q)$ forms on $X$ by $\Lambda^{p, q}(X, \mathbb{C})$.

Lemma 3.8. In the same setting as Theorem A, use the notations $\widehat{\omega}$ and $\widehat{\alpha}_{1}, \ldots, \widehat{\alpha}_{m-p-q+1}$ to denote a Kähler metric and smooth m-positive and semipositive forms in the corresponding classes, then for any smooth form $\widehat{\Phi} \in \Lambda^{p, q}(X, \mathbb{C})$ such that its class $\{\widehat{\Phi}\} \in P^{p, q}(X, \mathbb{C})$, there is a smooth form $\widehat{F}$ such that

$$
\widehat{\Omega} \wedge \widehat{\alpha}_{m-p-q+1} \wedge d d^{c} \widehat{F}=\widehat{\Omega} \wedge \widehat{\alpha}_{m-p-q+1} \wedge \widehat{\Phi} .
$$

Proof. Using Lemma 3.7, this follows from [DN13, Propositions 3.1, 3.2] (see also [DN06]).

Now we could prove Theorem A by using Lemma 3.8.

Proof of Theorem A. Assume that $\Phi \in P^{p, q}(X, \mathbb{C})$ and let $\widehat{\Phi}$ be a smooth representative of the class $\Phi$. Then by Lemma 3.8, there is a smooth form $\widehat{F}$ such that

$$
\widehat{\Omega} \wedge \widehat{\alpha}_{m-p-q+1} \wedge\left(\widehat{\Phi}-d d^{c} \widehat{F}\right)=0 .
$$

Thus $\widehat{\Phi}-d d^{c} \widehat{F}$ is a primitive $(p, q)$ form with respect to $\widehat{\Omega} \wedge \widehat{\alpha}_{m-p-q+1}$.

Applying Theorem 3.1, we have

$$
c \widehat{\Omega} \wedge\left(\widehat{\Phi}-d d^{c} \widehat{F}\right) \wedge \overline{\left(\widehat{\Phi}-d d^{c} \widehat{F}\right)} \geq 0
$$


at every point. By Stokes formula,

$$
Q(\Phi, \Phi)=c \int \widehat{\Omega} \wedge\left(\widehat{\Phi}-d d^{c} \widehat{F}\right) \wedge \overline{\left(\widehat{\Phi}-d d^{c} \widehat{F}\right)} \geq 0,
$$

where $c=i^{q-p}(-1)^{(p+q)(p+q+1) / 2}$.

Moreover, if $Q(\Phi, \Phi)=0$, then we have equalities everywhere. In particular, (9) is an equality at every point. By Theorem 3.1 again, this yields

$$
\widehat{\Phi}-d d^{c} \widehat{F}=0
$$

on $X$. Thus $\Phi=0$ in $H^{p, q}(X, \mathbb{C})$. This finishes the proof of the global HRR.

As an immediate consequence, we get the global HL. We only need to check that

$$
\Omega: H^{p, q}(X, \mathbb{C}) \rightarrow H^{n-q, n-p}(X, \mathbb{C})
$$

is injective. Assume that $\Phi \in H^{p, q}(X, \mathbb{C})$ satisfyies $\Omega \cdot \Phi=0$, then $\Phi$ is primitive and $Q(\Phi, \Phi)=0$. By HRR, $\Phi=0$, which finishes the proof of the HL.

Finally, the global LD follows from similar arguments as Lemma 3.6.

This finishes the proof of Theorem A.

\section{Further REMARKS}

4.1. Abstract Hodge-Riemann forms. In [DN13], Dinh-Nguyên gave an abstract version of HRR on compact Kähler manifolds by introducing the notion of Hodge-Riemann forms. By Theorem A, a more general abstract mixed HRR can be established.

In the sequel, the $\alpha_{j}$ are assumed to have the same positivity as above, i.e., $m$-positivity and semipositivity.

Definition 4.1. (analogous to [DN13, Definition 2.1]) A real $(k, k)$ form $\Omega \in \Lambda^{k, k}, k=n-p-q$, is called a Lefschetz form for the bidegree $(p, q)$ if the map

$$
\Omega: \Lambda^{p, q} \rightarrow \Lambda^{n-q, n-p}, \Phi \mapsto \Omega \wedge \Phi
$$

is an isomorphism. Assume $p+q \leq m \leq n$, a real $(k, k)$ form is said to be a Hodge-Riemann form for the bidegree $(p, q)$ if there is a continuous deformation $\Omega_{t} \in \Lambda^{k, k}$ with $0 \leq t \leq 1, \Omega_{0}=\Omega$ and $\Omega_{1}=\omega^{n-m} \wedge \alpha_{1} \wedge \ldots \wedge \alpha_{m-p-q}$ such that

$$
\Omega_{t} \wedge \alpha_{m-p-q+1}^{2 r} \text { is a Lefschetz form for the bidegree }(p-r, q-r)
$$

for every $r=0,1$ and $0 \leq t \leq 1$.

Definition 4.2. Let $X$ be a compact Kähler manifold of dimension $n$. Then $\Omega \in H^{k, k}(X, \mathbb{R})$ is called a Hodge-Riemann class, if it has a representative which is a Hodge-Riemann form at every point.

Analogous to [DN13], we have:

Theorem 4.3. Let $X$ be a compact Kähler manifold of dimension n, and let $\Omega$ be a Hodge-Riemann class. Then the HRR holds with respect to $\Omega$, where the primitive space is given by $\Omega \cdot \alpha_{m-p-q+1}$.

4.2. Generalized semi-small maps. Let $f: X \rightarrow Y$ be a proper surjective holomorphic map between two complex spaces. For every integer $k$ define

$$
Y^{k}=\left\{y \in Y \mid \operatorname{dim} f^{-1}(y)=k\right\} .
$$

The spaces $Y^{k}$ are analytic subvarieties of $Y$, whose disjoint union is $Y$.

Recall that $f$ is called a semi-small map in the sense of Goresky-MacPherson if

$$
\operatorname{dim} Y^{k}+2 k \leq \operatorname{dim} X
$$

for every $k \leq \operatorname{dim} X / 2$. Note that a semi-small map must be generically finite.

Semi-small maps can be generalized as follows. 
Definition 4.4. We call a proper surjective holomorphic map $f: X \rightarrow Y$ relatively semi-small if

$$
\operatorname{dim} Y^{k}+2 k \leq 2 \operatorname{dim} X-\operatorname{dim} Y
$$

for every $k$. Equivalently, $f$ is relatively semi-small if and only if there are no irreducible analytic subvarieties $T \subset X$ such that

$$
2 \operatorname{dim} T-2 \operatorname{dim} X+\operatorname{dim} Y>\operatorname{dim} f(T) .
$$

In particular, when $\operatorname{dim} X=\operatorname{dim} Y$, we get a semi-small map.

In [dCM02], a line bundle is called lef if the Kodaira map of its multiple induces a semi-small map. Corresponding to relatively semi-small maps, this can be generalized as follows.

Definition 4.5. A line bundle $L$ on a projective manifold $X$ is called relatively lef if $k L$ is generated by its global sections for some positive integer $k$ and the corresponding morphism

$$
\phi_{|k L|}: X \rightarrow Y=\phi_{|k L|}(X)
$$

is a relatively semi-small map.

Analogous to [dCM02], it is easy to get the following result.

Proposition 4.6. Let $f: X \rightarrow Y$ be a surjective holomorphic map from a compact Kähler manifold of dimension $n$ to a projective variety $Y$ of dimension $m$. Let $\omega$ be a Kähler class on $X$, and let $A_{1}, \ldots, A_{m-p-q}$ be ample line bundles on $Y$. If the relative $H L$ holds with respect to

$$
\omega^{n-m} \cdot f^{*} A_{1} \cdot \ldots \cdot f^{*} A_{m-p-q}
$$

for any $0 \leq p, q \leq p+q \leq m$, then $f$ is a relatively semi-small map.

Proof. Otherwise, assume that $f$ is not relatively semi-small. Then there is an irreducible analytic subvariety $T \subset X$ such that

$$
2 \operatorname{dim} T-2 n+m>\operatorname{dim} f(T) .
$$

Let $\{T\} \in H^{n-\operatorname{dim} T, n-\operatorname{dim} T}(X, \mathbb{R})$ be the fundamental class of $T$. The class $f^{*} A_{1} \cdot \ldots \cdot f^{*} A_{m-2(n-\operatorname{dim} T)}$ can be represented by an analytic cycle that does not intersect $T$, thus its intersection with $\{T\}$ is zero. In particular,

$$
\omega^{n-m} \cdot f^{*} A_{1} \cdot \ldots \cdot f^{*} A_{m-2(n-\operatorname{dim} T)} \cdot\{T\}=0
$$

which implies that the relative HL does not hold for the bidegree $(n-\operatorname{dim} T, n-\operatorname{dim} T)$.

This finishes the proof.

Remark 4.7. In the beautiful paper [dCM02], de Cataldo-Migliorini proved that $L$ is lef on a projective manifold of dimension $n$ if and only if the HL holds with respect to $L^{n-p-q}$ for every $0 \leq p, q \leq p+q \leq n$. Moreover, they proved that for semi-small maps, the deep Decomposition Theorem of Beilinson, Bernstein, Deligne and Gabber [BBD82] is equivalent to the non-degeneracy of certain intersection forms (i.e., HRR) associated with a stratification. They applied this result to give a new proof of the Decomposition Theorem for the direct image of the constant sheaf.

In our setting, we expect that $\phi_{|k L|}: X \rightarrow Y=\phi_{|k L|}(X)$ is relatively semi-small if and only if the relative HL holds with respect to $\omega^{n-m} \cdot L^{m-p-q}$ for every $0 \leq p, q \leq p+q \leq m$. We intend to discuss it elsewhere. Furthermore, we expect that the generalized form of Corollary A might be applied to study the topology of these maps.

\section{REFERENCES}

[AHK18] Karim Adiprasito, June Huh, and Eric Katz, Hodge theory for combinatorial geometries, Ann. of Math. (2) 188 (2018), no. 2, 381-452. MR 3862944

[BBD82] A. A. Bẹlinson, J. Bernstein, and P. Deligne, Faisceaux pervers, Analysis and topology on singular spaces, I (Luminy, 1981), Astérisque, vol. 100, Soc. Math. France, Paris, 1982, pp. 5-171. MR 751966

[BS02] Bo Berndtsson and Nessim Sibony, The $\bar{\partial}$-equation on a positive current, Invent. Math. 147 (2002), no. 2, 371-428. MR 1881924

[Cat08] Eduardo Cattani, Mixed Lefschetz theorems and Hodge-Riemann bilinear relations, Int. Math. Res. Not. IMRN (2008), no. 10, Art. ID rnn025, 20. MR 2429243

[dCM02] Mark Andrea A. de Cataldo and Luca Migliorini, The hard Lefschetz theorem and the topology of semismall maps, Ann. Sci. École Norm. Sup. (4) 35 (2002), no. 5, 759-772. MR 1951443 
[Dem12] Jean-Pierre Demailly, Complex analytic and differential geometry. online book, available at www-fourier. ujfgrenoble. fr/ demailly/manuscripts/agbook. pdf, Institut Fourier, Grenoble (2012).

[DN06] Tien-Cuong Dinh and Viêt-Anh Nguyên, The mixed Hodge-Riemann bilinear relations for compact Kähler manifolds, Geom. Funct. Anal. 16 (2006), no. 4, 838-849.

[DN13] Tien-Cuong Dinh and Viêt-Anh Nguyên, On the Lefschetz and Hodge-Riemann theorems, Illinois J. Math. 57 (2013), no. 1, 121-144. MR 3224564

[DS04] Tien-Cuong Dinh and Nessim Sibony, Groupes commutatifs d'automorphismes d'une variété kählérienne compacte, Duke Math. J. 123 (2004), no. 2, 311-328. MR 2066940

[DS05] - Green currents for holomorphic automorphisms of compact Kähler manifolds, J. Amer. Math. Soc. 18 (2005), no. 2, 291-312. MR 2137979

[EW14] Ben Elias and Geordie Williamson, The Hodge theory of Soergel bimodules, Ann. of Math. (2) 180 (2014), no. 3, 1089-1136. MR 3245013

[Gar59] Lars Garding, An inequality for hyperbolic polynomials, J. Math. Mech. 8 (1959), 957-965. MR 0113978

[Gro90] Misha Gromov, Convex sets and Kähler manifolds, Advances in Differential Geometry and Topology, ed. F. Tricerri, World Scientific, Singapore (1990), 1-38.

[Hor94] Lars Hormander, Notions of convexity, Progress in Mathematics, vol. 127, Birkhäuser Boston, Inc., Boston, MA, 1994. MR 1301332

[McM93] Peter McMullen, On simple polytopes, Invent. Math. 113 (1993), no. 2, 419-444. MR 1228132

[Tim98] V. A. Timorin, Mixed Hodge-Riemann bilinear relations in a linear context, Funktsional. Anal. i Prilozhen. 32 (1998), no. 4, 63-68, 96. MR 1678857

[Tim99] _ An analogue of the Hodge-Riemann relations for simple convex polyhedra, Uspekhi Mat. Nauk 54 (1999), no. 2(326), 113-162. MR 1711255

[Voi07] Claire Voisin, Hodge theory and complex algebraic geometry. I, english ed., Cambridge Studies in Advanced Mathematics, vol. 76, Cambridge University Press, Cambridge, 2007, Translated from the French by Leila Schneps. MR 2451566

[Wil16] Geordie Williamson, The Hodge theory of the Hecke category, arXiv preprint, arXiv:1610.06246 (2016).

[Xia18] Jian Xiao, Hodge-index type inequalities, hyperbolic polynomials and complex Hessian equations, arXiv preprint, arXiv:1810.04662 (2018).

Tsinghua University, Beijing, China

Email: jianxiao@mail.tsinghua.edu.cn 